\title{
NOTES SUR LES IXODIDAE DU GENRE HYALOMMA KOCH
}

\section{II. - HYALOMMA SCHULZEI OLENEV 1931}

\author{
Par Louis DELPY
}

Hyalomma schulzei Olenev 1931, est très probablement l'espèce la plus nettement caractérisée du genre Hyalomma. Le mâle, en effet, présente des stigmates du type « femelle », c'est-à-dire en triangle court plus ou moins arrondi, avec une queue extrêmement courte. Ce caractère, très net, ne se retrouve, à notre connaissance, chez aucun autre Hyalomma (fig. 3).

Olenev a donné une bonne description de l'espèce et trois figures. Il indique comme habitat le Nord et l'Ouest de l'Iran, mais l'hôte lui est inconnu.

En 1935, nous avons trouvé, sur deux lots de tiques provenant d'Ispahan et de Kachan (Iran), douze mâles, et une femelle à jeun, qui, à un premier examen, nous parurent être des Hyalomma dromedarii géants. Toutes les tiques avaient été récoltées sur des chameaux. L'un des mâles mesurait $9 \times 8 \mathrm{~mm}$., 5 , taille et prøportions véritablement anormales pour des mâles de Hyalomma et qui n'ont jamais été signalées jusqu'à présent. En Iran, les plus grands mâles que nous ayons vus appartenaient à l'espèce dromedarii et ne dépassaient pas $7 \mathrm{~mm}$.

Olenev indique comme dimensions de l'espèce schulzei, $6,5 \times 3,5$ chez le mâle et $6,5 \times 3 \mathrm{chez}$ la femelle, ce qui s'écarte à peine des dimensions de $H$. dromedarii ou de $H$. detritum. Néanmoins il nous suffit de noter la forme des stigmates du mâle, pour pouvoir rattacher nos tiques géantes à l'espèce schulzei.

Depuis cette épogue, nous n'avons pu nous procurer de spécimens vivants; il semble donc que cette tique soit relativement rare. D'autre part, en comparant nos spécimens à la description d'Olenev, il nous paraît évident que l'anteur russe n'a pu examiner que des individus relativement petits et non gorgés. En décrivant nos tiques géantes nous pensons établir quelques caractéristiques importantes de cette intéressante espèce.

Annales de Parasitologie, $\mathrm{T}$. XV, $\mathrm{N}^{\circ} 5 .-1^{\mathrm{er}}$ septembre 1937 , p. 419-430. 
Nous suivrons ici la méthode descriptive, adoptée dans notre travail de 1936 , pour l'étude de $H$. dromedarii. On verra d'ailleurs

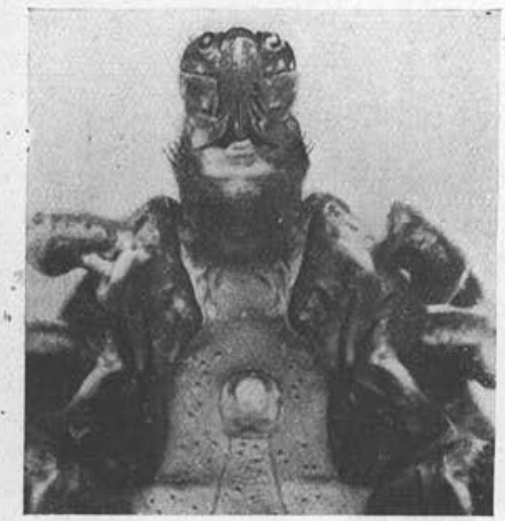

Fic. 1. - Hyalomma schulzei. Mâle, face ventrale. Capitulum et hanches $\mathrm{I}$.

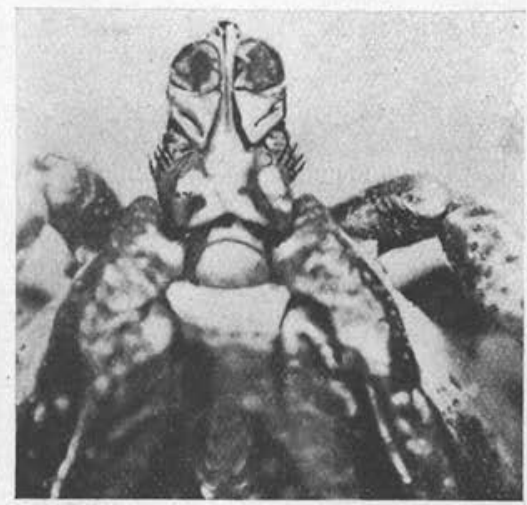

FiG. 2. - Hyalomma schulzei. Mâle, face dorsale. Capitulum, scapula, patte I.

que les deux espèces (schülzei et dromedarii) présentent une certaine similitude.

\section{MALE}

\section{Mensurations}

\begin{tabular}{|c|c|c|c|}
\hline LoNgUEUR $\times$ LARgeUR & SPÉCIMENS A JEUN & Spícimens gorgís & D'APRĖs OLENEv (1) \\
\hline Corps.. $\left\{\begin{array}{l}\text { Maxima ... } \\
\text { Minima ... } \\
\text { Moyenne.. }\end{array}\right.$ & $\begin{array}{c}8 \times 4,5 \mathrm{~mm} \\
6,5 \times 3,5 \mathrm{~mm} \\
7,4 \times 4 \mathrm{~mm}\end{array}$ & $\begin{array}{c}9 \times 8,5 \mathrm{~mm} \\
6,5 \times 4,5 \mathrm{~mm} \\
7,7 \times 6 \mathrm{~mm}\end{array}$ & $6,5 \times 3,5 \mathrm{~mm}$ \\
\hline Scutum. $\left\{\begin{array}{l}\text { Maxima... } \\
\text { Minima... } \\
\text { Moyenne.. }\end{array}\right.$ & $\begin{array}{l}7 \times 4,5 \mathrm{~mm} \\
5,5 \times 3,5 \mathrm{~mm} \\
6,4 \times 4 \mathrm{~mm} .\end{array}$ & $\begin{array}{l}7,8 \times 4,5 \mathrm{~mm} \\
5,5 \times 3,5 \mathrm{~mm} \\
6,7 \times 4,5 \mathrm{~mm}\end{array}$ & \\
\hline
\end{tabular}

(1) Chiffres donnés par Olenev ou établis d'après ses figures. 
2. Rapports des longueurs aUX largeurs des principales structures

\begin{tabular}{|c|c|c|c|c|}
\hline & Mixisum & Misimum & MOYENAE & $\begin{array}{l}\text { D'après } \\
\text { OLENEV (1) }\end{array}$ \\
\hline Corps $(L / 1) \ldots \ldots \ldots \ldots$ & 2 (à jeun) & 1,03 (gorgée) & 1,83 & 1,86 \\
\hline Scutum ............. & 1,75 & 1,50 & 1,62 & 1,62 \\
\hline Capitulum............. & 1,49 & 1,27 & 1,38 & 1,39 \\
\hline Base du capitulum..... & 0,61 & 0,56 & 0,58 & 0,53 \\
\hline Palpes .............. & 2 & 1,78 & 1,90 & 2 \\
\hline Article II (palpes)...... & 1 & 0,91 & 0,97 & 1,1 \\
\hline Article III (palpes)..... & 0,98 & 0,83 & 0,90 & 1 \\
\hline $\begin{array}{l}\text { Stigmate (queue com- } \\
\text { prise } \ldots \ldots \ldots \ldots \ldots \ldots\end{array}$ & & & 1,50 & 1,42 \\
\hline Tarse IV.............. & & & 2,60 & \\
\hline
\end{tabular}

3. Rapports des longueurs respectives des principales Structures

\begin{tabular}{|c|c|c|c|c|}
\hline & Maximum & Miximua & Moyenne & $\begin{array}{l}\text { D'APRÉS } \\
\text { OLENEV (1) }\end{array}$ \\
\hline 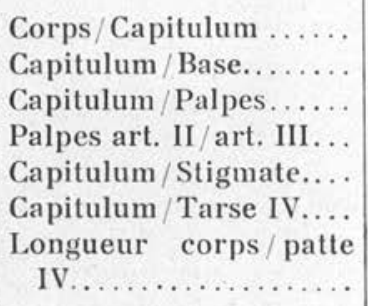 & $\begin{array}{l}5,70 \\
2,50 \\
1,90 \\
1,21\end{array}$ & $\begin{array}{c}5 \\
2,15 \\
1,74 \\
1\end{array}$ & $\begin{array}{c}5,30 \\
2,38 \\
1,82 \\
1,12 \\
1,65 \\
1 \\
1\end{array}$ & $\begin{array}{c}5,30 \\
2,60 \\
1,78 \\
1\end{array}$ \\
\hline
\end{tabular}

Ces chiffres permettent de juger à quel point la réplétion peut modifier l'aspect des mâles. Le mâle à jeun, surtout s'il est de petite taille, rappelle beaucoup $H$. dromedarii. C'est une tique allongée, plate, sans marge apparente. Le corps 's'élargit régulièrement depuis les scapulæ jusqu'au niveau des pattes III. Puis les bords latéraux restent à peu près parallèles et le bord postérieur est régulièrement arrondi. Il n'existe naturellement aucune constriction au niveau des stigmates, qui sont invisibles dorsalement.

(1) Chiffres donnés par Olenev ou établis d'après ses figurcs. 
Le mâle gorgé est parfois presque aussi large que long. L'abdomen distendu forme deux flancs rebondis, ce qui, dans certains cas, double presque la largeur de la tique. La largeur maxima est située un peu en avant des hanches IV, et non pas en arrière,



FiG. 3. - Hyalomma schulzei. Mâle. Stigmate droit, un écusson anal, 2 peltx.

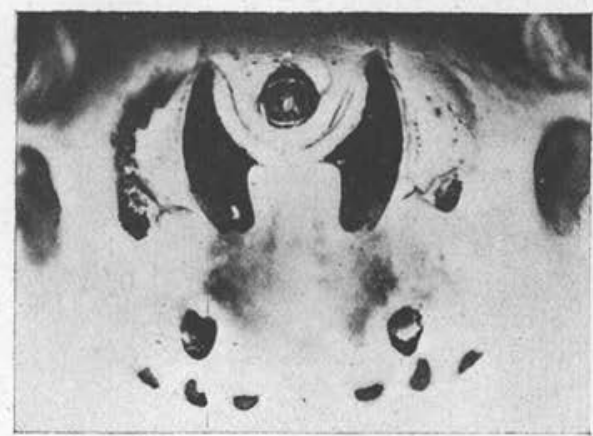

Fig. 4. - Hyalomma schulzei. Mâle, anus, écussons anaux, adanaux, subanaux, pelta.

comme chez $H$. dromedarii. Quant à l'épaisseur dorso-ventrale, elle atteint son maximum au niveau de l'anus, et peut atteindre $3 \mathrm{~mm}$., 5. Il existe, par conséquent, une véritable « face postérieure et ventrale » qui s'étend depuis la ligne anus-stigmates, jusqu'au bord postérieur du scutum, et qui porte les écussons de l'anus (fig. 4).

Scutum(fig. 5 et 7). - Teinte variant du brun roux foncé au brun noir [couleurs 113, 686, 706, du C.U.C. (1)]. Sillons complets et nets les cervicaux, larges et profonds, ridés, se prolongent en arrière jusqu'aux latéraux qui les unissent aux paramédians postérieurs. Le scutum est donc parcouru dans sa longueur par deux «fossæ» en vallon. Dans la moitié postérieure, le sillon médian postérieur débute au dos d'âne et va s'élargissant jusqu'au parma. Les sillons paramédians accessoires sont, surtout sur les sujets repus, bien dessinés, et forment deux triangles allongés, à fond ridé, convergents vers l'arrière. Ces trois sillons postérieurs limitent deux champs paramédians renflés et terminés postérieurement par les festons 1 . Les sillons marginaux sont très peu marqués, souvent constitués par une série de ponctuations.

(1) Par ces lettres : C.U.C., nous désignons le « Code universel des couleurs», de Seguy, édité par Lechevalier, Paris. 
Les ponctuations fines, -sont uniformément réparties sur tout le scutum et peu visibles. Par contre les fossettes pilifères, grandes et profondes, constituent des groupes très apparents sur les scapulæ, le champ cervical, les bords latéraux et les reliefs paramédians postérieurs. En outre, la partie médiane du scutum est, dans sa moitié antérieure, chagrinée par des stries à direction longitudinale, tandis que la partie postérieure porte des stries à direction

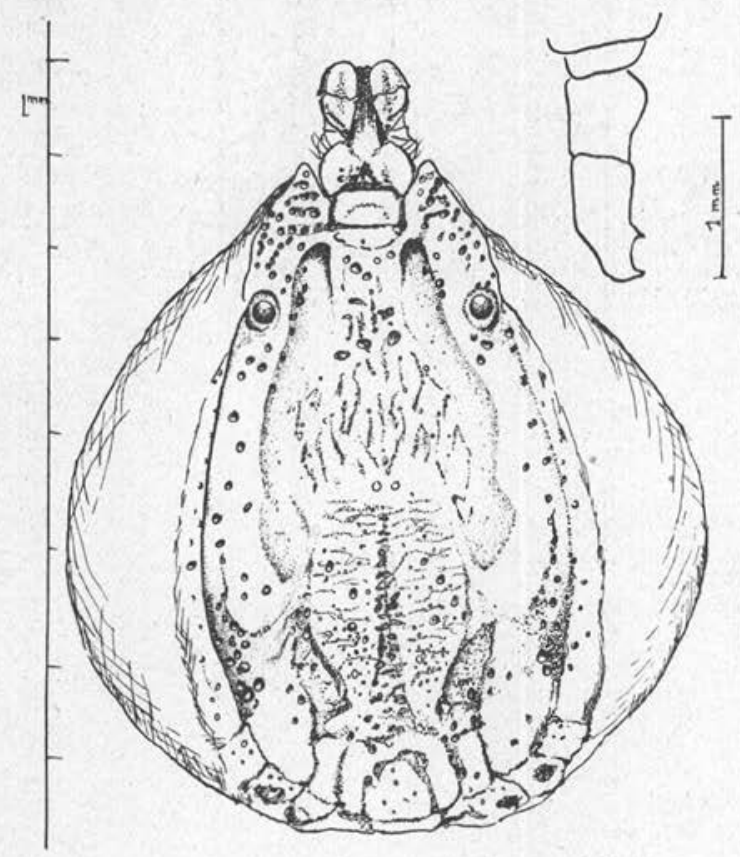

Fig. 5. - Hyalomma schulzei. Mâle gorgé. Vue dorsale et tarse IV.

transversale. Il en résulte un aspect très particulier, que l'on retrouve d'ailleurs sur la figure donnée par Olenev.

Le bord postérieur présente une large échancrure quadrangulaire, parfois limitée par un "pont » et qui encadre le parma. Ce dernier a, comme de règle, la même couleur que la face ventrale, c'est dire qu'en l'occurence il peut être blanc ou plus ou moins brun.

Les festons 1 et 2 sont bien séparés, allongés et étroits. Les festons 3,4 et 5 sont fusionnés et constituent le lobe postéro-latèral, peu saillant. Sur la face postéro-ventrale de la tique, exislent 10 pelta, qui ne sont bien visibles que chez les sujets à ventre blanc. 
Face ventrale. - Couleur allant du blanc au brun foncé. Parties chitineuses de la même teinte que le scutum. Hanches sans particularités notables, si ce n'est la tubérosité bien développée à la branche interne de la hanche 1 . Système anal complet; les écussons sub-anaux sont situés chez les sujets à jeun dans le pro-

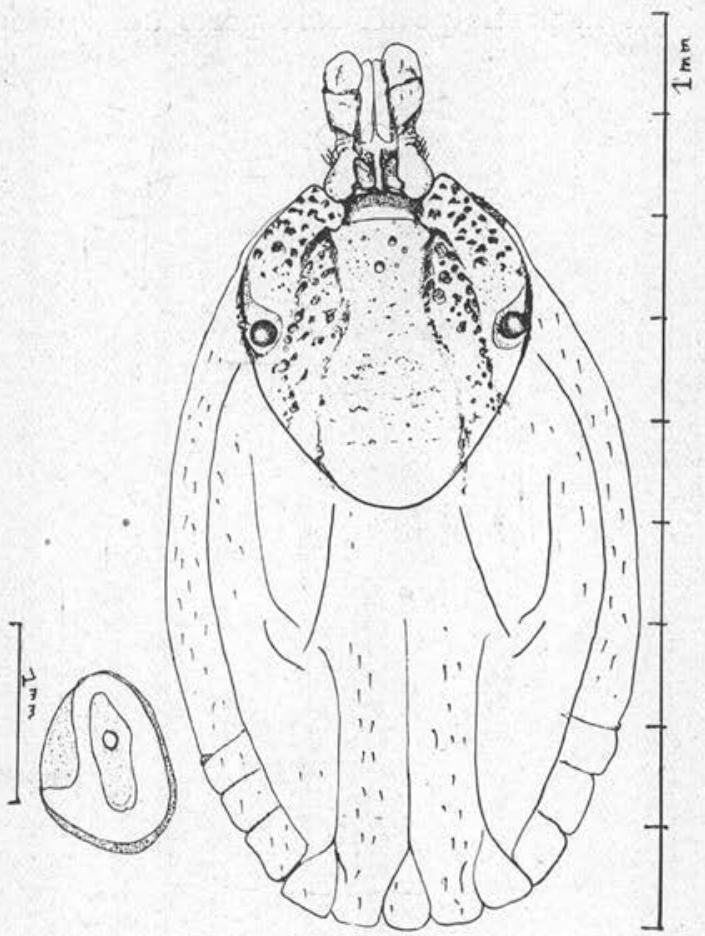

Fig. 6. - Hyalomma schulzei. Femelle à jeun. Vue dorsale et stigmate.

longement des anaux. Ils sont tirés vers l'extérieur chez les sujets gorgés (figs 4 et 8 ).

Les stigmates sont, rappelons-le, du type femelle, ovoïdes avec une queue très courte (fig. 3 ).

Capitulum (figs $1,2,5,7$ et $9, a-b-c-d$ ). - Le capitulum parait très court. En réalité, les mensurations prouvent que, comme chez $H$. dromedarii, sa longueur est à peu près égale au $1 / 5$ de la longueur totale. Par contre, il est très large, massif. Les chiffres donnés plus haut montrent que les palpes sont très courts; ils sont 


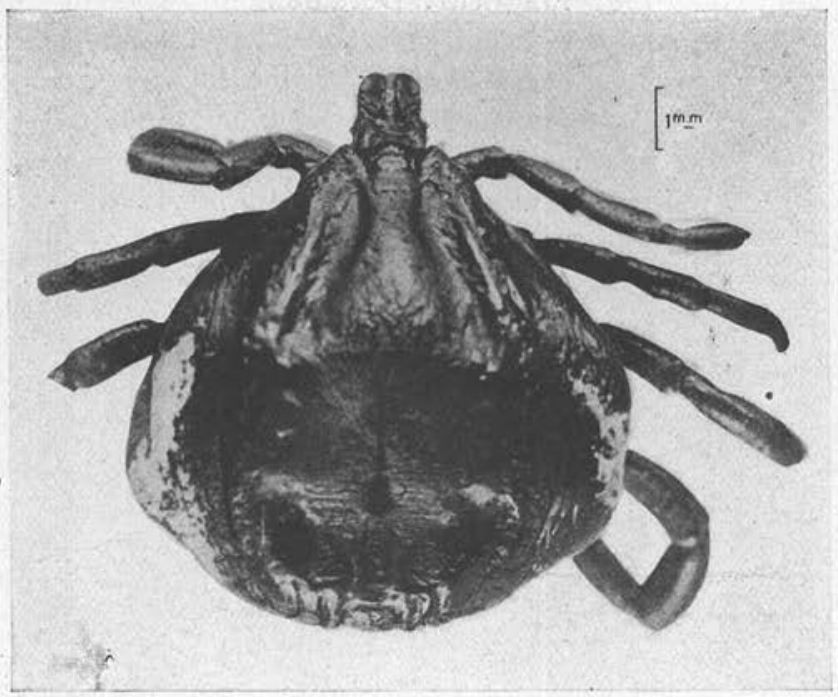

FıG. 7. - Hyalomma schulzei. Mâle, face dorsale. Spécimen gorgé.

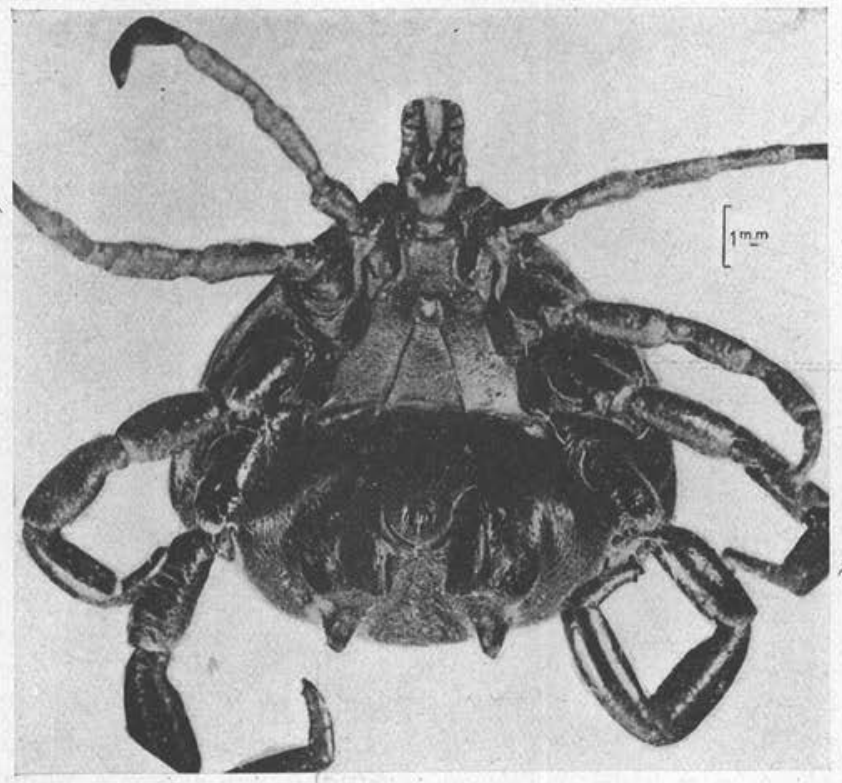

FıG, 8, - Hyalomma schulzei. Mâle, face ventrale. Spécimen gorgé. 
excavés sur toute leur longueur. Dorsalement les articles 2 et 3 sont subégaux, presque aussi larges que longs. La base du capitulum, à peu près deux fois plus large que longue, est vigoureusement modelée. Les cornua nettes donnent au bord postérieur un aspect cintré. On remarquera sur les figs 5 et 7 que la partie proximale des palpes est assez éloignée du bord antérieur de la
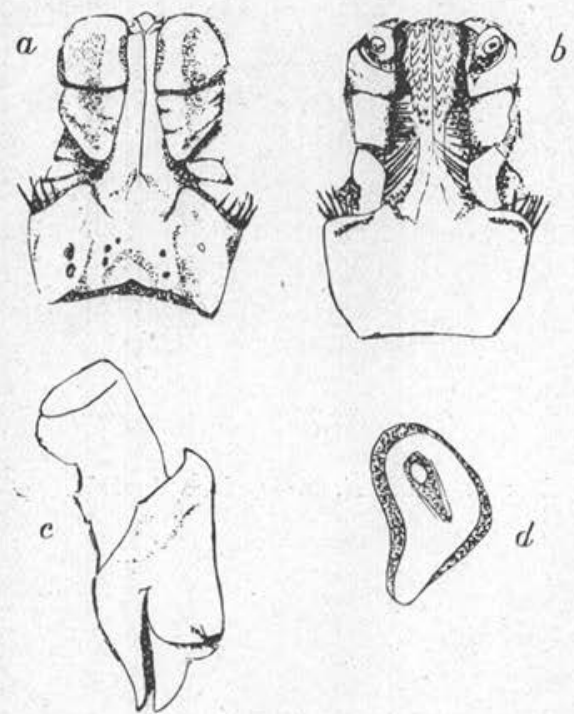

Fig. 9. - Hyalomma schulzei, Mâle. - $a$, Capitulum, face dorsale ; $b$, Capitulum, face ventrale; $c$, Hanche I, droite ; $d$, Stigmate.

base, et ceci explique que le capitulum ait une longueur normale, malgré la brièveté des palpes et de la base. Le palpiger porte une collerette de fortes et longues soies à pointe antérieure (fig. 5).

Les palpes sont nettement plus clairs que la base. L'article II est brun-jaune (193, C.U.C.), l'article III un peu plus foncé.

A la face inférieure, les articles II et III sont égaux. L'apophyse en croissant de l'article I est très développée et porte de longues et fortes soies. Hypostome en massue, dentition $3 / 3$.

Pattes. - De plus en plus fortes de I à IV. Patte IV aussi longue que le corps, tarse IV épais. La couleur, sur les pattes postérieures est brun-roux, avec une ligne dorsale et l'extrémité distale des articles nettement blanches. L'importance du blanc aug- 
mente sur les pattes antérieures, qui présentent parfois l'aspect caractéristique des pattes de $H$. lusitanicum (blanc avec taches brunes). On distingue sur la photographie fig. 2 cet aspect bien caractérisé à la patte antérieure droite. Comme toujours, la dessiccation accentue le contraste des teintes.

\section{Femelle}

Nous n'avons malheureusement qu'un spécimen qui soit certainement une femelle de $H$. schulzei. La détermination des femelles de Hyalomma est généralement difficile et il y a toujours intérêt, lorsque la chose est possible, à baser les descriptions sur des spécimens d'élevage. N'ayant pu avoir de femelles vivantes, nous ne retenons qu'un spécimen à jeun, qui, par sa taille, ses proportions et les rides caractéristiques du scutum se rapproche plus de l'espèce schulzei que d'aucune autre. Cette femelle fut d'ailleurs trouvée avec 5 mâles à jeun, sur un chameau de Kachan.

\section{Mensurations}

Nous donnons à titre de comparaison les mensurations d'après le texte et la figure d'Olenev (1931).

1. Corps : $8 \times 4 \mathrm{~mm}$. - Olenev donne : $6,5 \times 3 \mathrm{~mm}$.

2. Scutum et capitulum : $3 \mathrm{~mm}$., 85 .

3. Largeur du scutum : 2 mm., 85 .

4. Rapports des longueurs aUx Largeurs des principales STruCtures

Olenev

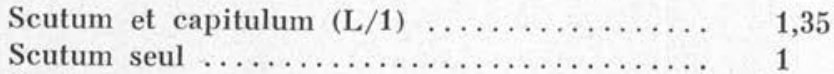

1,5

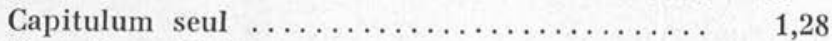

1,1

Base du capitulum $\ldots \ldots \ldots \ldots \ldots \ldots \ldots \ldots \ldots$

0,43

1,38



2,40

0,46

Palpes Art. II $\ldots \ldots \ldots \ldots \ldots \ldots \ldots \ldots \ldots \ldots \ldots$

Palpes Art. III

1,40

2,50

Stigmate

1

1,50

Tarse 
5. RAPPoRTS DES LONGUEURS RESPECTIVES DES PRINCIPALES STRUCTURES

Olenev

Scutum/capitulum .....................

Capitulum/base .......................

2,15

Capitulum/palpes $\ldots \ldots \ldots \ldots \ldots \ldots \ldots \ldots \ldots \ldots$

Palpes Art. II/Art. III $\ldots \ldots \ldots \ldots \ldots \ldots \ldots \ldots$

Capitulum/stigmates $\ldots \ldots \ldots \ldots \ldots \ldots \ldots \ldots$

Capitulum/tarses.$\ldots \ldots \ldots \ldots \ldots \ldots \ldots \ldots \ldots$

Scutum/distance des scapulæ aux yeux ......

2,95

1,90

1,50

3

1,40

1,80

1,80

0,90

2,35

1,50

2,05

Notre femelle (fig. 6) se distingue d'abord des femelles d'autres espèces du genre, par sa taille. Même chez $H$. dromedarii, qui est une grande tique, les femelles à jeun n'ont pas plus de $7 \mathrm{~mm}$. Nous retenons donc ce caractère, qu'Olenev ne mentionne pas, puisque son (ou ses) spécimens n'a (ou n'ont) que $6 \mathrm{~mm}$., 5 .

Le corps est d'un ovale bien régulier, de couleur brun foncé (116, C.U.C.). Le système des sillons sur le conscutum est complet, le médian postérieur profond et net va de la base du scutum au bord postérieur, où il se termine en fourche, délimitant ainsi le feston médian. Le sillon marginal est également très net. Il borde les festons sur toute leur longueur et atteint en avant le bord du scutum, au-dessous des yeux. Les festons sont au nombre de 11, soit 5 de chaque côté du médian. Face ventrale de la même couleur que le dos. Sillons nets. Un peu en avant de l'anus une dépression transversale.

Stigmates du type habituel chez les Hyalomma femelles, avec très courte queue coudée à angle droit. Hanche I présentant, comme chez le mâle une protubérance nette sur la branche interne.

Scutum. - Aussi large que long. Les yeux sont plus près de la pointe des scapulæ que du bord postérieur. Ils, sont très noirs, très saillants, sphériques. La largeur maxima est au niveau des yeux.

Les sillons cervicaux, très profonds, sculptés par des fossettes grandes et parfois confluentes, sont larges et s'ouvrent au bord postérieur. Les scapulæ sont saillantes, couvertes de grandes fossettes. Le champ cervical, plus clair que l'écusson (146, C.U.C.) est régulièrement garni de fines ponctuations. En outre, des fossettes assez nombreuses sont éparses. La surface présente assez nettement les rides transversales signalées chez le mâle. 
Capitulum. - Moins long que la moitié du scutum, large et massif. Les palpes, plus longs que chez le mâle, à angles très arrondis, présentent dorsalement une surface assez régulièrement bombée, avec une dépression intéressant les parties contiguës des articles II et III. Le bord postérieur de l'art. II est taillé en biseau. L'article III est arrondi à son extrémité distale. Le bord interne de chaque palpe est légèrement et régulièrement convexe.

La base, dont la largeur est plus de deux fois plus grande que la longueur, présente deux aires poreuses profondes, à axes parallèles, séparées par une crête rectiligne bien marquée. Les parties latérales sont renflées en olive, les cornua sont peu accusées, et une dépression étroite relie les aires poreuses au bord postérieur. La base est de même teinte que le champ cervical du scutum, avec les angles plus foncés. Les palpes sont brun clair (191 C.U.C.), l'art. II et l'art. III, de même couleur. Collerette de soies présente à la base du palpiger.

Ventralement, les articles II et III sont égaux, leur bord externe régulièrement convexe. Apophyse sétifère en croissant de l'article I moins développée que chez le mâle.

Pattes longues et fortes. L'ornementation est du type annelé, la ligne dorsale claire peu apparente.

On peut se rendre compte que cette femelle présente peu de caractères propres à assurer la détermination. Il nous est arrivé de mettre en élevage une grosse femelle gorgée $(26 \times 11 \mathrm{~mm}$.) qui nous paraissait appartenir à l'espèce schulzei, et de constater, par l'examen de sa descendance qu'elle appartenait en réalité à l'espèce dromedarii. Nous pensons qu'on peut différencier ces deux espèces : 1) par la taille de la femelle à jeun ; 2) par les proportions du capitulum qui est plus court chez $H$. schulzei ; 3) par la position des yeux, qui sont plus rapprochés des scapulæ chez schulzei.

Nous ne sommes pas convaincu que le scutum et le capitulum figurés par Olenev se rapportent réellement à $H$. schulzei. Le capitulum est relativement très long : le rapport scutum-capitulum qui est de 2,15 pour notre femelle n'est que de 1,90 pour celle d'Olenev, ce qui la rapproche de $H$. dromedarii dont le même rapport est de 1,8. D'autre part, sur la figure donnée par Olenev, les yeux sont à égale distance de la pointe des scapulæ et du bord postérieur, au lieu d'être légèrement antérieurs. 


\section{RÉSUMÉ}

La description de l'espèce Hyalomma schulzei Olenev 1931, est complétée par les résultats de l'étude de 12 mâles et d'une femelle à jeun, trouvés sur des chameaux. Nous insistons particulièrement sur les dimensions des mâles, qui font de l'espèce $H$. schulzei, la plus grande qui ait été décrite jusqu'ici dans le genre. Nous montrons les modifications importantes apportées par la réplétion à l'aspect des mâles.

Enfin l'étude détaillée de la femelle montre qu'elle est assez difficilement différenciable de $H$. dromedarii. Il est possible que la femelle représentée par Olenev (1931) appartienne à cette dernière espèce.

Un mâle gorgé et un mâle à jeun, mesurant respectivement $8 \times 7 \mathrm{~mm}$., 2 et $8 \times 4 \mathrm{~mm}$., 5 sont déposés à l'Institut de Parasitologie de la Faculté de médecine de Paris (Professeur E. Brumpt). Les autres spécimens sont conservés à Hessarek.

\section{BiBLIOGRAPHIE}

Delpy (L.). - Notes sur les Ixodoidea du genre Hyalomma. Annales de Parasitologie, XIV, 1936, p. 205.

OLEnev. - Les tiques parasites Ixodoidea, de la faune de IU.R.S.S., Leningrad, Ed. de l'Ac. des Sciences (en russe), 1931.

Service des recherches vétérinaires de l'Empire iranien. Hessarek par Karadj, Téhéran (Iran). 